TRANSACTIONS OF THE

AMERICAN MATHEMATICAL SOCIETY

Volume 356, Number 12, Pages 4839-4855

S 0002-9947(04)03667-0

Article electronically published on June 25, 2004

\title{
GLOBAL STRICHARTZ ESTIMATES FOR SOLUTIONS TO THE WAVE EQUATION EXTERIOR TO A CONVEX OBSTACLE
}

\author{
JASON L. METCALFE
}

\begin{abstract}
In this paper, we show that certain local Strichartz estimates for solutions of the wave equation exterior to a convex obstacle can be extended to estimates that are global in both space and time. This extends the work that was done previously by H. Smith and C. Sogge in odd spatial dimensions. In order to prove the global estimates, we explore weighted Strichartz estimates for solutions of the wave equation when the Cauchy data and forcing term are compactly supported.
\end{abstract}

\section{INTRODUCTION}

The purpose of this paper is to show that certain local Strichartz estimates for solutions to the wave equation exterior to a nontrapping obstacle can be extended to estimates that are global in both space and time. In [12, Smith and Sogge proved this result for odd spatial dimensions $n \geq 3$. Here, we extend this result to all spatial dimensions $n \geq 3$.

If $\Omega$ is the exterior domain in $\mathbb{R}^{n}$ to a compact obstacle and $n \geq 3$ is an even integer, we are looking at solutions to the following wave equation:

$$
\left\{\begin{array}{l}
\square u(t, x)=\partial_{t}^{2} u(t, x)-\Delta u(t, x)=F(t, x), \quad(t, x) \in \mathbb{R} \times \Omega, \\
u(0, x)=f(x) \in \dot{H}_{D}^{\gamma}(\Omega), \\
\partial_{t} u(0, x)=g(x) \in \dot{H}_{D}^{\gamma-1}(\Omega), \\
u(t, x)=0, \quad x \in \partial \Omega .
\end{array}\right.
$$

Here $\Omega$ is the complement in $\mathbb{R}^{n}$ to a compact set contained in $\{|x| \leq R\}$ with $C^{\infty}$ boundary. Moreover, $\Omega$ is nontrapping in the sense that there is a $T_{R}$ such that no geodesic of length $T_{R}$ is completely contained in $\{|x| \leq R\} \cap \Omega$. The case $\Omega=\mathbb{R}^{n}$ is permitted.

We say that $1 \leq r, s \leq 2 \leq p, q \leq \infty$ and $\gamma$ are admissible if the following two estimates hold.

Local Strichartz estimates. For $f, g, F(t, \cdot)$ supported in $\{|x| \leq R\}$, solutions to (1.1) satisfy

$$
\begin{aligned}
\|u\|_{L_{t}^{p} L_{x}^{q}([0,1] \times \Omega)}+\sup _{0 \leq t \leq 1}\|u(t, \cdot)\|_{H_{D}^{\gamma}(\Omega)}+\sup _{0 \leq t \leq 1}\left\|\partial_{t} u(t, \cdot)\right\|_{H_{D}^{\gamma-1}(\Omega)} \\
\quad \leq C\left(\|f\|_{H_{D}^{\gamma}(\Omega)}+\|g\|_{H_{D}^{\gamma-1}(\Omega)}+\|F\|_{L_{t}^{r} L_{x}^{s}([0,1] \times \Omega)}\right) .
\end{aligned}
$$

Received by the editors November 14, 2002.

2000 Mathematics Subject Classification. Primary 35L05. 
Global Minkowski Strichartz estimates. In the case when $\Omega=\mathbb{R}^{n}$, solutions to (1.1) satisfy

$$
\begin{aligned}
& \|u\|_{L_{t}^{p} L_{x}^{q}\left(\mathbb{R}^{1+n}\right)}+\sup _{t}\|u(t, \cdot)\|_{\dot{H}^{\gamma}\left(\mathbb{R}^{n}\right)}+\sup _{t}\left\|\partial_{t} u(t, \cdot)\right\|_{\dot{H}^{\gamma-1}\left(\mathbb{R}^{n}\right)} \\
& \quad \leq C\left(\|f\|_{\dot{H}^{\gamma}\left(\mathbb{R}^{n}\right)}+\|g\|_{\dot{H}^{\gamma-1}\left(\mathbb{R}^{n}\right)}+\|F\|_{L_{t}^{r} L_{x}^{s}\left(\mathbb{R}^{1+n}\right)}\right) .
\end{aligned}
$$

Additionally, for technical reasons we need to assume $2>r$ and $\gamma \leq(n-1) / 2$.

The global Minkowski Strichartz estimate (1.3) is a generalization of the work of Strichartz [15, 16]. The local Strichartz estimates (1.2) for solutions to the homogeneous $(F=0)$ wave equation in a domain exterior to a convex obstacle were established by Smith and Sogge in [13. In [12, Smith and Sogge demonstrated that a lemma of Christ and Kiselev 2] (see also [12] for a proof) could be used to establish local estimates for solutions to the nonhomogeneous problem.

While the arguments that follow are valid in any domain exterior to a nontrapping obstacle, it is not currently known whether the local Strichartz estimates (1.2) hold if the obstacle is not convex. Related eigenfunction estimates are, however, known to fail if $\partial \Omega$ has a point of convexity.

We note here that $p, q, r, s, \gamma$ are admissible in the above sense if the obstacle is convex, $n \geq 3$,

$$
\begin{gathered}
q, s^{\prime}<\frac{2(n-1)}{n-3} ; \quad \frac{1}{p}+\frac{n}{q}=\frac{n}{2}-\gamma=\frac{1}{r}+\frac{n}{s}-2 ; \\
\frac{1}{p}=\left(\frac{n-1}{2}\right)\left(\frac{1}{2}-\frac{1}{q}\right) ; \quad \frac{1}{r^{\prime}}=\left(\frac{n-1}{2}\right)\left(\frac{1}{2}-\frac{1}{s^{\prime}}\right)
\end{gathered}
$$

where $r^{\prime}, s^{\prime}$ represent the conjugate exponents to $r, s$ respectively. In particular, notice that we have admissibility in the conformal case

$$
p, q=\frac{2(n+1)}{n-1} ; \quad r, s=\frac{2(n+1)}{n+3} ; \quad \gamma=\frac{1}{2} .
$$

Additionally, we note that it is well known (see, e.g., 4) that in the homogeneous case $(F=0)$ the global Minkowski Strichartz estimate (1.3) holds if and only if $n \geq 2,2 \leq p \leq \infty, 2 \leq q<\infty, \gamma=\frac{n}{2}-\frac{n}{q}-\frac{1}{p}$, and

$$
\frac{2}{p} \leq \frac{n-1}{2}\left(1-\frac{2}{q}\right) \text {. }
$$

Thus, (1.4) provides a necessary condition for admissibility.

The main result of this paper states that for such a set of indices a similar global estimate holds for solutions to the wave equation in the exterior domain.

Theorem 1.1. Suppose $n \geq 3$. If $p, q, r, s, \gamma$ are admissible and $u$ is a solution to the Cauchy problem (1.1), then

$$
\|u\|_{L_{t}^{p} L_{x}^{q}(\mathbb{R} \times \Omega)} \leq C\left(\|f\|_{\dot{H}_{D}^{\gamma}(\Omega)}+\|g\|_{\dot{H}_{D}^{\gamma-1}(\Omega)}+\|F\|_{L_{t}^{r} L_{x}^{s}(\mathbb{R} \times \Omega)}\right) .
$$

Throughout the sequel, we will focus on the case $n \geq 4$. The techniques herein can be modified to handle the $n=3$ case, but since this case was previously handled by Smith and Sogge [12] and since this complicates the exposition, we choose not to provide these details.

The key differences between the general case and the odd dimensional case are the lack of the strong Huygens' principle and the fact that the local energy no 
longer decays exponentially. Local energy decay and the homogeneous Sobolev spaces $\dot{H}_{D}^{\gamma}(\Omega)$ will be discussed in more detail in the next section.

This paper is organized as follows. In the next section, we will discuss the homogeneous Sobolev spaces and the local decay of energy. In the third section, we will establish our main estimates in Minkowski space. These include certain weighted Strichartz estimates for the homogeneous wave equation with compactly supported data in free space. In the fourth section, we give an alternate proof of a mixed norm estimate of Smith and Sogge [12] which is valid in all spatial dimensions. Finally, in Section 5, we prove Theorem 1.1.

At the final stage of preparation, we learned that N. Burq [1] has independently obtained the results from this paper using a slightly different method.

It is a pleasure to thank C. Sogge for his guidance and patience during this study. The author would also like to thank the referee for several helpful suggestions.

\section{ENERGy EStimates}

We begin here with a few notes on the homogeneous Sobolev spaces $\dot{H}_{D}^{\gamma}(\Omega)$. The homogeneous Sobolev norms, $\dot{H}^{\gamma}\left(\mathbb{R}^{n}\right)$, are given by

$$
\|f\|_{\dot{H}^{\gamma}\left(\mathbb{R}^{n}\right)}=\left\|(\sqrt{-\Delta})^{\gamma} f\right\|_{L^{2}\left(\mathbb{R}^{n}\right)} .
$$

For functions supported on a fixed compact set, the homogeneous Sobolev space $\dot{H}^{\gamma}\left(\mathbb{R}^{n}\right)$ is comparable to the inhomogeneous Sobolev space $H^{\gamma}\left(\mathbb{R}^{n}\right)$. Moreover, for $\gamma<\frac{n}{2}$, the homogeneous Sobolev spaces $\dot{H}^{\gamma}\left(\mathbb{R}^{n}\right)$ are preserved under multiplication by smooth cutoff functions.

Fixing a smooth cutoff function $\beta \in C_{c}^{\infty}$ such that $\beta(x) \equiv 1$ for $|x| \leq R$, for $|\gamma|<n / 2$, we are able to define

$$
\|f\|_{\dot{H}_{D}^{\gamma}(\Omega)}=\|\beta f\|_{\dot{H}_{D}^{\gamma}(\tilde{\Omega})}+\|(1-\beta) f\|_{\dot{H}^{\gamma}\left(\mathbb{R}^{n}\right)}
$$

where $\tilde{\Omega}$ is a compact manifold with boundary containing $B_{R}=\Omega \cap\{|x| \leq R\}$. In particular, notice that for functions (or distributions) supported in $\{|x| \leq R\}$, we have $\|f\|_{\dot{H}_{D}^{\gamma}(\Omega)}=\|f\|_{\dot{H}_{D}^{\gamma}(\tilde{\Omega})}$.

Functions $f \in \dot{H}_{D}^{\gamma}(\tilde{\Omega})$ satisfy the Dirichlet condition $\left.f\right|_{\partial \tilde{\Omega}}=0$ (when this makes sense). With the Dirichlet condition fixed, we may define the spaces $\dot{H}_{D}^{\gamma}(\tilde{\Omega})$ in terms of eigenfunctions of $\Delta$. Since $\tilde{\Omega}$ is compact, we have an orthonormal basis of $L^{2}(\tilde{\Omega}),\left\{u_{j}\right\} \subset H_{D}^{1}(\tilde{\Omega}) \cap C^{\infty}(\tilde{\Omega})$ with $\Delta u_{j}=-\lambda_{j} u_{j}$ where $0<\lambda_{j} \nearrow \infty$. Thus, for $\gamma \geq 0$, it is natural to define

$$
\dot{H}_{D}^{\gamma}(\tilde{\Omega})=\left\{v \in L^{2}(\tilde{\Omega}): \sum_{j \geq 0}|\hat{v}(j)|^{2} \lambda_{j}^{\gamma}<\infty\right\}
$$

where $\hat{v}(j)=\left(v, u_{j}\right)$. The $\dot{H}_{D}^{\gamma}(\tilde{\Omega})$ norm is given by

$$
\|v\|_{\dot{H}_{D}^{\gamma}(\tilde{\Omega})}^{2}=\sum_{j}|\hat{v}(j)|^{2} \lambda_{j}^{\gamma} .
$$

Defining $\dot{H}_{D}^{\gamma}(\tilde{\Omega})$ for $\gamma<0$ in terms of duality, it is not difficult to see that the above characterization for the norm also holds for negative $\gamma$. Additionally, we mention that

$$
\|v\|_{\dot{H}_{D}^{1}(\tilde{\Omega})}^{2}=\left\|v^{\prime}\right\|_{L^{2}(\tilde{\Omega})}^{2}
$$


and for $r<s$,

$$
\|v\|_{\dot{H}_{D}^{r}(\tilde{\Omega})}^{2} \leq C\|v\|_{\dot{H}_{D}^{s}(\tilde{\Omega})}^{2} .
$$

See, e.g., [18] for further details. Notice that by defining $\dot{H}_{D}^{\gamma}(\tilde{\Omega})$ in this way, it builds in the necessary compatibility conditions on the data. For example, when $\gamma \geq 2$, we must have that $\left.\Delta^{j} f\right|_{\partial \tilde{\Omega}}=0$ for $2 j \leq \gamma$.

At this point, we may define $H_{D}^{\gamma}(\Omega)$ similarly. That is, let

$$
\|f\|_{H_{D}^{\gamma}(\Omega)}=\|\beta f\|_{H_{D}^{\gamma}(\tilde{\Omega})}+\|(1-\beta) f\|_{H^{\gamma}\left(\mathbb{R}^{n}\right)} .
$$

Note that since $\lambda_{0}>0$, we have $\dot{H}_{D}^{\gamma}(\tilde{\Omega})=H_{D}^{\gamma}(\tilde{\Omega})$. Also, notice that for functions $u$ supported in $|x|<R,\|u\|_{H_{D}^{\gamma}(\Omega)} \approx\|u\|_{H_{D}^{\gamma}(\tilde{\Omega})}$.

One of the key results that will allow us to establish the global estimates from the local estimates and the global Minkowski estimates is local energy decay. It is this result that requires the nontrapping assumption on the obstacle. In odd dimensions, we are able to get exponential energy decay; see Taylor [17], Lax-Philips 5], Vainberg [19, Morawetz-Ralston-Strauss 10], Strauss [14, and Morawetz 8, 9 . In even spatial dimensions, the decay is significantly less. The version that we will use in this paper is

Local energy decay. For $n \geq 4$ even, data $f, g$ supported in $\{|x| \leq R\}, 0 \leq \gamma$, and $\beta(x)$ smooth, supported in $\{|x| \leq R\}$, there exists $C<\infty$ such that for solutions to (1.1) where $F=0$ the following holds:

$$
\|\beta u(t, \cdot)\|_{H_{D}^{\gamma}(\Omega)}+\left\|\beta \partial_{t} u(t, \cdot)\right\|_{H_{D}^{\gamma-1}(\Omega)} \leq C|t|^{-n / 2}\left(\|f\|_{H_{D}^{\gamma}(\Omega)}+\|g\|_{H_{D}^{\gamma-1}(\Omega)}\right) .
$$

This is a generalized version of the results of Melrose [6]. Before showing how we can derive this generalized version of local energy decay, we would like to mention here the related works of Morawetz [7, Ralston [11, and Strauss [14.

Notice that for $\gamma<n / 2$, the Sobolev norms in (2.1) may be replaced with the homogeneous Sobolev norms.

Proof of equation (2.1). By density, we may, without loss of generality, assume that $f, g$ are $C^{\infty}$. When $n \geq 4$ is even, Melrose [6] was able to show that a solution to the homogeneous $(F=0)$ Cauchy problem (1.1) outside a nontrapping obstacle with data $f, g$ supported in $\{|x| \leq R\}$ must satisfy

$$
\int_{B_{R}}|\nabla u(t, x)|^{2} d x+\int_{B_{R}}\left(\partial_{t} u(t, x)\right)^{2} d x \leq C t^{-n}\left(\int|\nabla f|^{2} d x+\int|g|^{2} d x\right)
$$

where $B_{R}=\{|x| \leq R\} \cap \Omega$. Since $u$ can be controlled locally by $\nabla_{x} u$, (2.2) implies

$$
\|\beta(\cdot) u(t, \cdot)\|_{H_{D}^{1}(\tilde{\Omega})}+\left\|\beta(\cdot) \partial_{t} u(t, \cdot)\right\|_{L^{2}(\tilde{\Omega})} \leq C t^{-n / 2}\left(\|f\|_{H_{D}^{1}(\tilde{\Omega})}+\|g\|_{L^{2}(\tilde{\Omega})}\right) .
$$

Since $\left[\square, \partial_{t}\right]=0$ and $\partial_{t}$ preserves the support of the data and the boundary condition, we have that $u_{t}(t, x)$ is a solution of

$$
\left\{\begin{array}{l}
\square u_{t}(t, x)=0, \quad(t, x) \in \mathbb{R} \times \Omega, \\
u_{t}(0, x)=g(x), \\
\partial_{t} u_{t}(0, x)=\Delta f(x), \\
u_{t}(t, x)=0, \quad x \in \partial \Omega .
\end{array}\right.
$$


Thus, by (2.2) and the fact that $\square u=0$, we have

$$
\begin{aligned}
\left\|\beta(\cdot) u_{t}(t, \cdot)\right\|_{H_{D}^{1}(\tilde{\Omega})}+\| \beta(\cdot) \Delta & u(t, \cdot) \|_{L^{2}(\tilde{\Omega})} \\
& =\left\|\beta(\cdot) u_{t}(t, \cdot)\right\|_{H_{D}^{1}(\tilde{\Omega})}+\left\|\beta(\cdot) u_{t t}(t, \cdot)\right\|_{L^{2}(\tilde{\Omega})} \\
& \leq C t^{-n / 2}\left(\|g\|_{H_{D}^{1}(\tilde{\Omega})}+\|\Delta f\|_{L^{2}(\tilde{\Omega})}\right) \\
& =C t^{-n / 2}\left(\|f\|_{H_{D}^{2}(\tilde{\Omega})}+\|g\|_{H_{D}^{1}(\tilde{\Omega})}\right) .
\end{aligned}
$$

Thus, by elliptic regularity, (2.3), and the monotonicity in $\gamma$ of the norms $\|\cdot\|_{H_{D}^{\gamma}(\tilde{\Omega})}$, we see that

$$
\|\beta(\cdot) u(t, \cdot)\|_{H_{D}^{2}(\tilde{\Omega})}+\left\|\beta(\cdot) \partial_{t} u(t, \cdot)\right\|_{H_{D}^{1}(\tilde{\Omega})} \leq C t^{-n / 2}\left(\|f\|_{H_{D}^{2}(\tilde{\Omega})}+\|g\|_{H_{D}^{1}(\tilde{\Omega})}\right) .
$$

If we look similarly at $u_{t t}, u_{t t t}$, etc., we see that

$$
\|\beta(\cdot) u(t, \cdot)\|_{H_{D}^{s}(\tilde{\Omega})}+\left\|\beta(\cdot) \partial_{t} u(t, \cdot)\right\|_{H_{D}^{s-1}(\tilde{\Omega})} \leq C t^{-n / 2}\left(\|f\|_{H_{D}^{s}(\tilde{\Omega})}+\|g\|_{H_{D}^{s-1}(\tilde{\Omega})}\right)
$$

for any positive integer $s$. By complex interpolation and the characterization of the Sobolev spaces given above, this yields (2.1) for any $\gamma \geq 1$.

We now work to obtain (2.1) with $\gamma<1$. To do so, let $\tilde{g}$ be the solution of

$$
\begin{cases}\Delta \tilde{g}(x)=g(x), & \text { in }\{|x| \leq R\} \cap \Omega, \\ \tilde{g}(x)=0, & \text { on }\{|x|=R\} \cup \partial \Omega .\end{cases}
$$

Fix a smooth cutoff function $\chi(x)$ with $\chi(x) \equiv 1$ on supp $g$ and supp $\chi \subset\{|x|<R\}$. Then

$$
\Delta(\chi \tilde{g})=g+\psi
$$

where $\psi \in C_{c}^{\infty}(\Omega)$ and, by elliptic regularity,

$$
\|\psi\|_{L^{2}(\Omega)} \leq C\|g\|_{H_{D}^{-1}(\Omega)} .
$$

If $v$ is the solution to

$$
\left\{\begin{array}{l}
\square v(t, x)=0, \\
v(0, x)=\chi(x) \tilde{g}(x), \\
\partial_{t} v(0, x)=f(x), \\
v(t, x)=0, \quad x \in \partial \Omega,
\end{array}\right.
$$

since $\left[\square, \partial_{t}\right]=0$, we have

$$
\left\{\begin{array}{l}
\square \partial_{t} v(t, x)=0, \\
\partial_{t} v(0, x)=f(x), \\
\partial_{t}^{2} v(0, x)=\Delta(\chi(x) \tilde{g}(x)), \\
\partial_{t} v(t, x)=0, \quad x \in \partial \Omega .
\end{array}\right.
$$


Thus, by (2.2) and (2.7), we have

$$
\begin{aligned}
\left\|\beta(\cdot) \partial_{t} v(t, \cdot)\right\|_{L^{2}(\tilde{\Omega})}+\| \beta(\cdot) & \partial_{t}^{2} v(t, \cdot) \|_{H_{D}^{-1}(\tilde{\Omega})} \\
& \leq C\|\beta(\cdot) \Delta v(t, \cdot)\|_{H_{D}^{-1}(\tilde{\Omega})}+\left\|\beta(\cdot) \partial_{t} v(t, \cdot)\right\|_{L^{2}(\tilde{\Omega})} \\
& \leq C\|\beta(\cdot) v(t, \cdot)\|_{H_{D}^{1}(\tilde{\Omega})}+\left\|\beta(\cdot) \partial_{t} v(t, \cdot)\right\|_{L^{2}(\tilde{\Omega})} \\
& \leq C t^{-n / 2}\left(\|\chi(x) \tilde{g}(x)\|_{H_{D}^{1}(\Omega)}+\|f\|_{L^{2}(\Omega)}\right) \\
& \leq C t^{-n / 2}\left(\|f\|_{L^{2}(\Omega)}+\|g\|_{H_{D}^{-1}(\Omega)}\right) .
\end{aligned}
$$

Since $u-\partial_{t} v$ also solves a homogeneous wave equation with $C_{c}^{\infty}(\Omega)$ data

$$
\left\{\begin{array}{l}
\square\left(u-\partial_{t} v\right)=0, \\
\left(u-\partial_{t} v\right)(0, \cdot)=0, \\
\left(\partial_{t} u-\partial_{t}^{2} v\right)(0, \cdot)=\psi, \\
\left(u-\partial_{t} v\right)(t, x)=0, \quad \text { for } x \in \partial \Omega,
\end{array}\right.
$$

we have

$$
\begin{aligned}
\| \beta(\cdot)\left(u-\partial_{t} v\right)(t, \cdot) & \left\|_{L^{2}(\tilde{\Omega})}+\right\| \beta(\cdot)\left(\partial_{t} u-\partial_{t}^{2} v\right)(t, \cdot) \|_{H_{D}^{-1}(\tilde{\Omega})} \\
& \leq\left\|\beta(\cdot)\left(u-\partial_{t} v\right)(t, \cdot)\right\|_{H_{D}^{1}(\tilde{\Omega})}+\left\|\beta(\cdot)\left(\partial_{t} u-\partial_{t}^{2} v\right)(t, \cdot)\right\|_{L^{2}(\tilde{\Omega})} \\
& \leq C t^{-n / 2}\|\psi\|_{L^{2}(\Omega)} \\
& \leq C t^{-n / 2}\|g\|_{H_{D}^{-1}(\Omega)} .
\end{aligned}
$$

Combining (2.8) and (2.9), it follows easily that

$$
\|\beta(\cdot) u(t, \cdot)\|_{L^{2}(\tilde{\Omega})}+\left\|\beta(\cdot) \partial_{t} u(t, \cdot)\right\|_{H_{D}^{-1}(\tilde{\Omega})} \leq C t^{-n / 2}\left(\|f\|_{L^{2}(\Omega)}+\|g\|_{H_{D}^{-1}(\Omega)}\right) .
$$

Finally, if we interpolate with (2.3), we see that we obtain (2.1) for $0 \leq \gamma \leq 1$, which completes the proof.

\section{Weighted Minkowski estimates}

In this section we show that weighted versions of the Minkowski Strichartz estimates for solutions to the homogeneous wave equation can be obtained when the initial data are compactly supported. Specifically, we are looking at the homogeneous free wave equation

$$
\left\{\begin{array}{l}
\square w(t, x)=\partial_{t}^{2} w(t, x)-\Delta w(t, x)=0, \quad(t, x) \in \mathbb{R} \times \mathbb{R}^{n}, \\
w(0, x)=f(x) \in H^{\gamma}\left(\mathbb{R}^{n}\right) \\
\partial_{t} w(0, x)=g(x) \in H^{\gamma-1}\left(\mathbb{R}^{n}\right)
\end{array}\right.
$$

where the Cauchy data $f, g$ are supported in $\left\{x \in \mathbb{R}^{n}:|x|<R\right\}$.

We begin by showing that one can obtain weighted versions of the energy inequality. Here we need only slightly modify the arguments of Hörmander [3] (Lemma 6.3.5, p. 101) and Lax-Philips [5] (Appendix 3). 
Lemma 3.1. Suppose that $n \geq 3$. Let $w(t, x)$ be a solution to the homogeneous Minkowski wave equation (3.1) with smooth initial data $f, g$ supported in $\{|x| \leq R\}$. Then the following estimate holds:

$$
\int(t-|x|)^{2}\left(\left|\nabla_{x} w(t, x)\right|^{2}+\left(\partial_{t} w(t, x)\right)^{2}\right) d x \leq C_{R}\left(\int|\nabla f|^{2}+|g|^{2} d x\right) .
$$

Proof. It is not difficult to check that

$$
\operatorname{div}_{x} p+\partial_{t} q=N(w) \square w
$$

where

$$
\begin{aligned}
N(w)= & 4 t(x \cdot \nabla w)+2\left(r^{2}+t^{2}\right) w_{t}+2(n-1) t w \\
p=-2 t w_{t}^{2} x-4 t(x \cdot \nabla w) \nabla w+2 t|\nabla w|^{2} x & \quad-2\left(r^{2}+t^{2}\right) w_{t} \nabla w-2(n-1) t w \nabla w \\
q= & 4 t(x \cdot \nabla w) w_{t}+\left(r^{2}+t^{2}\right)\left(|\nabla w|^{2}+w_{t}^{2}\right)+2(n-1) t w w_{t}-(n-1) w^{2} .
\end{aligned}
$$

If we integrate over a cylinder $[0, T] \times\left\{x \in \mathbb{R}^{n}:|x| \leq \bar{R}\right\}$ for $\bar{R}$ sufficiently large, Huygens' principle and the divergence theorem gives us that

$$
\int_{t=T} q d x-\int_{t=0} q d x=0 .
$$

Here, since the initial data are compactly supported, we have

$$
\begin{aligned}
\int_{t=0} q d x & =\int_{t=0} r^{2}\left(\left|\nabla_{x} w(0, x)\right|^{2}+w_{t}(0, x)^{2}\right)-(n-1) w(0, x)^{2} d x \\
& \leq C_{R}\left(\int|\nabla f|^{2}+|g|^{2} d x\right) .
\end{aligned}
$$

Now, let us introduce the standard invariant vector fields

$$
Z_{0}=t \partial_{t}+\sum_{j=1}^{n} x_{j} \partial_{j}, \quad Z_{0 k}=t \partial_{k}+x_{k} \partial_{t}, \quad Z_{j k}=x_{k} \partial_{j}-x_{j} \partial_{k}
$$

for $j, k=1,2, \ldots, n$. Notice that

$$
\int_{t=T} q d x=\int_{t=T}\left(\left|Z_{0} w\right|^{2}+\sum_{0 \leq j<k \leq n}\left|Z_{j k} w\right|^{2}+2(n-1) t w w_{t}-(n-1) w^{2}\right) d x .
$$

Applying Lemma 6.3.5 of Hörmander [3] (p. 101), we see that (3.2)-(3.4) yield

$$
\left\|Z_{0} w(t, \cdot)\right\|_{L^{2}\left(\mathbb{R}^{n}\right)}^{2}+\sum_{j<k}\left\|Z_{j k} w(t, \cdot)\right\|_{L^{2}\left(\mathbb{R}^{n}\right)}^{2} \leq C_{R}\left(\int|\nabla f|^{2}+|g|^{2} d x\right) .
$$

Thus, we see that in order to complete the proof, it suffices to show that

$$
\int(t-r)^{2}\left(w_{t}^{2}+|\nabla w|^{2}\right) d x \leq\left\|Z_{0} w\right\|_{L^{2}\left(\mathbb{R}^{n}\right)}^{2}+\sum_{0 \leq j<k \leq n}\left\|Z_{j k} w\right\|_{L^{2}\left(\mathbb{R}^{n}\right)}^{2} .
$$


Since the Cauchy-Schwarz inequality gives us that $|\nabla w| \geq w_{r}$ and since $4 \operatorname{tr} w_{t} w_{r}$ $\geq-2 \operatorname{tr}\left(w_{t}^{2}+w_{r}^{2}\right)$, we have

$$
\begin{aligned}
\left\|Z_{0} w\right\|_{L^{2}\left(\mathbb{R}^{n}\right)}^{2}+ & \sum_{0 \leq j<k \leq n}\left\|Z_{j k} w\right\|_{L^{2}\left(\mathbb{R}^{n}\right)}^{2}=\int\left(t^{2}+r^{2}\right)\left(w_{t}^{2}+|\nabla w|^{2}\right)+4 t r w_{t} w_{r} d x \\
& =\int(t-r)^{2}\left(w_{t}^{2}+|\nabla w|^{2}\right)+2 t r w_{t}^{2}+2 \operatorname{tr}|\nabla w|^{2}+4 t r w_{t} w_{r} \\
& \geq \int(t-r)^{2}\left(w_{t}^{2}+|\nabla w|^{2}\right)
\end{aligned}
$$

as desired.

Next, we look at the weighted analog of the dispersive inequality when the initial data have compact supports.

Lemma 3.2. Suppose $n \geq 2$. Let $w$ be a solution to the homogeneous Minkowski wave equation (3.1) with initial data $f, g$ supported in $\{|x| \leq R\}$. Then, we have

$$
\left\|(|t|-|x|)^{(n-1) / 2} w(t, x)\right\|_{L_{x}^{\infty}(\{|t|-|x| \geq 2 R\})} \leq \frac{C_{R}}{|t|^{(n-1) / 2}}\left(\|f\|_{L^{2}\left(\mathbb{R}^{n}\right)}+\|g\|_{L^{2}\left(\mathbb{R}^{n}\right)}\right),
$$

$$
\left\|(|t|-|x|)^{(n+1) / 2} \partial_{t} w(t, x)\right\|_{L_{x}^{\infty}(\{|t|-|x| \geq 2 R\})} \leq \frac{C_{R}}{|t|^{(n-1) / 2}}\left(\|f\|_{L^{2}\left(\mathbb{R}^{n}\right)}+\|g\|_{L^{2}\left(\mathbb{R}^{n}\right)}\right) .
$$

Additionally, for $n \geq 4$,

$$
\left\|(|t|-|x|)^{\theta} w(t, x)\right\|_{L_{x}^{2}(\{|t|-|x| \geq 2 R\})} \leq C_{R, \theta}\left(\|f\|_{L^{2}\left(\mathbb{R}^{n}\right)}+\|g\|_{L^{2}\left(\mathbb{R}^{n}\right)}\right)
$$

for any $\theta<1$.

We note that (3.8) holds for any $\theta<1 / 2$ when $n=3$. This is sufficient to yield the results in the sequel for $q>2$. Since, however, the $n=3$ case was handled by Smith and Sogge [12] and since this would complicate the argument, we choose not to provide the details here.

Proof. By scaling, we may assume that $R=1$. For simplicity, we will demonstrate the result for $t>0$.

Begin by writing $w=w_{1}+w_{2}$, where $w_{1}$ is a solution of the homogeneous Minkowski wave equation (3.1) with Cauchy data $\left.\left(w, w_{t}\right)\right|_{t=0}=(f, 0)$ and $w_{2}$ is a solution of the Minkowski wave equation (3.1) with Cauchy data $\left.\left(w, w_{t}\right)\right|_{t=0}=$ $(0, g)$. It will, thus, suffice to show that the estimate holds for $w_{1}$ and $w_{2}$ separately. Since the arguments are the same for each piece, we will restrict our attention to showing that the estimate holds for $w_{2}$, the more technical piece.

From equations (5.43) and (5.48) of [18] (p. 222), we have that

$$
w_{2}(t, x)=R(t, \cdot) * g
$$

where

$$
R(t, x)=\lim _{\varepsilon \searrow 0} c_{n} \operatorname{Im}\left(|x|^{2}-(t-i \varepsilon)^{2}\right)^{-(n-1) / 2} .
$$

Thus, since $g$ is supported in $\{|x|<1\}$, we can apply the Schwarz inequality to see $|w(t, x)| \leq C\left(\int_{|y| \leq 1}|R(t, x-y)|^{2} d y\right)^{1 / 2}\|g\|_{L^{2}\left(\mathbb{R}^{n}\right)} \leq C_{n} \sup _{|y| \leq 1}|R(t, x-y)|\|g\|_{L^{2}\left(\mathbb{R}^{n}\right)}$. 
Since $|x| \leq|t|-2$ and $|y| \leq 1$, we have that $t^{2}-|x-y|^{2} \approx t^{2}-|x|^{2}$. Thus, by (3.10), we have

$$
\sup _{|y| \leq 1}|R(t, x-y)| \leq C|t|^{-(n-1) / 2}(|t|-|x|)^{-(n-1) / 2},
$$

which completes the proof of (3.6). Since it is easy to see that

$$
\sup _{|y| \leq 1}\left|\partial_{t} R(t, x-y)\right| \leq C|t|^{-(n-1) / 2}(|t|-|x|)^{-(n+1) / 2},
$$

we also get (3.7).

For (3.8), we again use (3.9) and (3.10) to see that

$$
\begin{aligned}
& \left\|(|t|-|x|)^{\theta} w(t, x)\right\|_{L_{x}^{2}(\{|t|-|x| \geq 2\})} \\
& \quad \leq C\left\|(t-|x|)^{\theta} \int \frac{1}{\left(t^{2}-|x-y|^{2}\right)^{(n-1) / 2}} g(y) d y\right\|_{L^{2}(\{t-|x| \geq 2\})} .
\end{aligned}
$$

Since $|y| \leq 1$ and $t-|x| \geq 2$, we have that the right-hand side is controlled by

$$
C\left\|\int \frac{1}{(t+|x-y|)^{(n-1) / 2}(t-|x-y|)^{((n-1) / 2)-\theta}} g(y) d y\right\|_{L^{2}(\{t-|x| \geq 2\})} .
$$

By Young's inequality, this is dominated by

$$
C\left\|\frac{1}{(t+|x|)^{(n-1) / 2}} \frac{1}{(t-|x|)^{((n-1) / 2)-\theta}}\right\|_{L^{2}(\{t-|x| \geq 1\})}\|g\|_{1} .
$$

Since $g$ is compactly supported, by the Schwarz inequality, we have that $\|g\|_{1} \leq$ $C\|g\|_{2}$. We thus want to examine the $L^{2}$ norm of the kernel above. Writing this in polar coordinates, we see that the square of this norm is bounded by

$$
\int_{0}^{t-1} \int_{S^{n-1}} \frac{1}{(t-\rho)^{n-1-2 \theta}} d \sigma(\omega) d \rho
$$

This establishes (3.8) since this integral is bounded independent of $t$ for $n \geq 4$ and any $\theta<1$.

From the previous two lemmas, we are able to derive a weighted Strichartz estimate for solutions to the Minkowski wave equation with compactly supported initial data.

Theorem 3.3. Suppose $n \geq 4$ and $p, q, \gamma$ are admissible. Let $w$ be a solution to the homogeneous Minkowski wave equation (3.1) with Cauchy data $f, g$ supported in $\{|x| \leq R\}$. Then, for any $\theta<1$, we have the following estimate:

$$
\left\|(|t|-|x|)^{\theta} w(t, x)\right\|_{L_{t}^{p} L_{x}^{q}\left(\mathbb{R}^{1+n}\right)} \leq C_{R}\left(\|f\|_{H^{\gamma}\left(\mathbb{R}^{n}\right)}+\|g\|_{H^{\gamma-1}\left(\mathbb{R}^{n}\right)}\right) .
$$

Proof. By the global Minkowski Strichartz estimate (1.3) and finite propogation speed, it will suffice to show the estimate in the case $|t|-|x| \geq 2 R$. We will, also, stick to the case $t \geq 0$. Let $S_{t}=\{x: t-|x| \geq 2 R\}$.

By Lemma 3.1 and Lemma 3.2, we have

$$
\begin{aligned}
\left\|(t-|x|)^{\theta} \partial_{t} w(t, x)\right\|_{L_{x}^{2}\left(S_{t}\right)} & \leq C\left(\|f\|_{H^{1}\left(\mathbb{R}^{n}\right)}+\|g\|_{L^{2}\left(\mathbb{R}^{n}\right)}\right) \\
\left\|(t-|x|)^{\theta} \partial_{t} w(t, x)\right\|_{L_{x}^{\infty}\left(S_{t}\right)} & \leq \frac{C}{t^{(n-1) / 2}}\left(\|f\|_{H^{1}\left(\mathbb{R}^{n}\right)}+\|g\|_{L^{2}\left(\mathbb{R}^{n}\right)}\right) .
\end{aligned}
$$


In the second inequality, we have used the monotonicity in $\gamma$ of $H^{\gamma}$. By RieszThorin interpolation, we have

$$
\left\|(t-|x|)^{\theta} \partial_{t} w(t, x)\right\|_{L_{x}^{q}\left(S_{t}\right)} \leq C\left(\frac{1}{t^{(n-1) / 2}}\right)^{\left(1-\frac{2}{q}\right)}\left(\|f\|_{H^{1}\left(\mathbb{R}^{n}\right)}+\|g\|_{L^{2}\left(\mathbb{R}^{n}\right)}\right) .
$$

Since by (1.4)

$$
p \cdot \frac{n-1}{2}\left(1-\frac{2}{q}\right)>\frac{p}{2}\left(\frac{n-1}{2}\left(1-\frac{2}{q}\right)\right) \geq 1,
$$

we see that taking the $L_{t}^{p}$ norm of both sides yields

$$
\left\|(t-|x|)^{\theta} \partial_{t} w(t, x)\right\|_{L_{t}^{p} L_{x}^{q}\left(\{t \geq 2 R\} \times S_{t}\right)} \leq C\left(\|f\|_{H^{1}\left(\mathbb{R}^{n}\right)}+\|g\|_{L^{2}\left(\mathbb{R}^{n}\right)}\right)
$$

for $n \geq 3$.

Similarly, we may interpolate between (3.6) and (3.8) to see that

$$
\left\|(t-|x|)^{\theta} w(t, x)\right\|_{L_{t}^{p} L_{x}^{q}\left(\{t \geq 2 R\} \times S_{t}\right)} \leq C\left(\|f\|_{L^{2}\left(\mathbb{R}^{n}\right)}+\|g\|_{L^{2}\left(\mathbb{R}^{n}\right)}\right)
$$

for $n \geq 4$.

If we now argue as we did in obtaining (2.10) from (2.3), we see that (3.11) and (3.12) yield

$$
\left\|(t-|x|)^{\theta} w(t, x)\right\|_{L_{t}^{p} L_{x}^{q}\left(\{t \geq 2 R\} \times S_{t}\right)} \leq C\left(\|f\|_{L^{2}\left(\mathbb{R}^{n}\right)}+\|g\|_{\dot{H}^{-1}\left(\mathbb{R}^{n}\right)}\right) .
$$

The result, then, follows from the monotonicity of the Sobolev norms.

\section{Mixed estimates in Minkowski SPaCE}

In this section, as in Smith and Sogge [12], we collect a couple of results that follow from the fact that

$$
\sup _{\xi}|\xi|^{2 \gamma}\left[\int|\widehat{\beta}(\xi-\eta)| \delta(\tau-|\eta|) d \eta\right] \leq C_{n, \gamma, \beta} \tau^{2 \gamma}
$$

if $\beta$ is a smooth function supported in $\{|x| \leq 1\}$ and $0 \leq \gamma \leq \frac{n-1}{2}$.

The first of these results is Lemma 2.2 of [12].

Lemma 4.1. Let $\beta$ be a smooth function supported in $\{|x| \leq 1\}$. Suppose $0 \leq \gamma \leq$ $\frac{n-1}{2}$. Then

$$
\int_{-\infty}^{\infty}\left\|\beta(\cdot) e^{i t \sqrt{-\Delta}} f(\cdot)\right\|_{\dot{H}^{\gamma}\left(\mathbb{R}^{n}\right)}^{2} d t \leq C_{n, \gamma, \beta}\|f\|_{\dot{H}^{\gamma}\left(\mathbb{R}^{n}\right)}^{2} .
$$

The second result is an analog of a result in [12] that was shown in odd spatial dimensions. Here we require a different argument that does not rely on the sharp Huygens' principle.

Lemma 4.2. Let $w$ be a solution to the Cauchy problem for the Minkowski wave equation

$$
\left\{\begin{array}{l}
\square w(t, x)=\partial_{t}^{2} w(t, x)-\Delta w(t, x)=F(t, x), \quad(t, x) \in \mathbb{R} \times \mathbb{R}^{n}, \\
w(0, x)=f(x), \\
\partial_{t} w(0, x)=g(x) .
\end{array}\right.
$$


Suppose that the global Minkowski Strichartz estimate (1.3) holds, that $0 \leq \gamma \leq \frac{n-1}{2}$, and that $r<2$. Then, for $\beta$ a smooth function supported in $\{|x| \leq 1\}$, we have

$$
\begin{aligned}
\sup _{|\alpha| \leq 1} & \int_{-\infty}^{\infty}\left\|\beta(\cdot) \partial_{x}^{\alpha} w(t, \cdot)\right\|_{\dot{H}^{\gamma-1}\left(\mathbb{R}^{n}\right)}^{2} d t \\
& \leq C\left(\|f\|_{\dot{H}^{\gamma}\left(\mathbb{R}^{n}\right)}+\|g\|_{\dot{H}^{\gamma-1}\left(\mathbb{R}^{n}\right)}+\|F\|_{L_{t}^{r} L_{x}^{s}\left(\mathbb{R}^{1+n}\right)}\right)^{2} .
\end{aligned}
$$

Proof. If $F=0$, the result follows from Lemma 4.1. Thus, it will suffice to show that

$$
\int_{0}^{\infty}\|\beta(\cdot) w(t, \cdot)\|_{\dot{H}^{\gamma}\left(\mathbb{R}^{n}\right)}^{2} d t \leq C\|F\|_{L_{t}^{r} L_{x}^{s}\left(\mathbb{R}^{1+n}\right)}^{2}
$$

when the initial data $f, g$ are assumed to vanish.

We begin by establishing that

$$
T F(t, x)=\Lambda^{\gamma} \beta(\cdot) \int \frac{\sin (t-s) \Lambda}{\Lambda} F(s, \cdot) d s
$$

is bounded from $L_{t}^{r} L_{x}^{s}\left(\mathbb{R}_{+}^{1+n}\right)$ to $L_{t}^{2} L_{x}^{2}\left(\mathbb{R}^{1+n}\right)$. In other words, we want to show that

$$
\int\left\|\beta(\cdot) \int \frac{\sin (t-s) \Lambda}{\Lambda} F(s, \cdot) d x\right\|_{\dot{H}^{\gamma}\left(\mathbb{R}^{n}\right)}^{2} d t \leq C\|F\|_{L_{t}^{r} L_{x}^{s}\left(\mathbb{R}^{1+n}\right)}^{2}
$$

when $F$ is assumed to vanish for $t<0$.

By Strichartz estimate (1.3), we have

$$
\begin{aligned}
\int \frac{|\eta|^{2 \gamma}}{|\eta|^{2}}|\tilde{F}(|\eta|, \eta)|^{2} d \eta & =\int \frac{|\eta|^{2 \gamma}}{|\eta|^{2}}\left|\int e^{-i s|\eta|} \hat{F}(s, \eta) d s\right|^{2} d \eta \\
& \leq \sup _{t} \int|\eta|^{2 \gamma}\left|\int_{0}^{t} \frac{e^{i(t-s)|\eta|}}{|\eta|} \hat{F}(s, \eta) d s\right|^{2} d \eta \\
& \leq \sup _{t}\left(\|w(t, \cdot)\|_{\dot{H}^{\gamma}\left(\mathbb{R}^{n}\right)}^{2}+\left\|\partial_{t} w(t, \cdot)\right\|_{\dot{H}^{\gamma-1}\left(\mathbb{R}^{n}\right)}^{2}\right) \\
& \leq C\|F\|_{L_{t}^{r} L_{x}^{s}\left(\mathbb{R}^{1+n}\right)}^{2}
\end{aligned}
$$

where $\tilde{F}$ denotes the space-time Fourier transform of $F$.

By Plancherel's theorem in $t, x$, we have

$$
\begin{aligned}
\int \| \beta(\cdot) \int \frac{e^{i(t-s) \Lambda}}{\Lambda} & F(s, \cdot) d s \|_{\dot{H}^{\gamma}\left(\mathbb{R}^{n}\right)}^{2} d t \\
& =\int_{0}^{\infty} \int|\xi|^{2 \gamma}\left|\int \hat{\beta}(\xi-\eta) \delta(\tau-|\eta|) \frac{1}{|\eta|} \tilde{F}(|\eta|, \eta) d \eta\right|^{2} d \xi d \tau .
\end{aligned}
$$

By the Schwarz inequality in $\eta$, this can be bounded by

$$
\begin{aligned}
\int_{0}^{\infty} \int|\xi|^{2 \gamma}\left(\int|\hat{\beta}(\xi-\eta)|\right. & \delta(\tau-|\eta|) d \eta) \\
& \times\left(\int|\hat{\beta}(\xi-\eta)| \delta(\tau-|\eta|) \frac{1}{|\eta|^{2}}|\tilde{F}(|\eta|, \eta)|^{2} d \eta\right) d \xi d \tau
\end{aligned}
$$


Applying (4.1), (4.3), and Young's inequality, we see that

$$
\begin{aligned}
\int \| \beta(\cdot) & \int \frac{e^{i(t-s) \Lambda}}{\Lambda} F(s, \cdot) d s \|_{\dot{H}^{\gamma}\left(\mathbb{R}^{n}\right)}^{2} d t \\
& \leq \int_{0}^{\infty} \int \tau^{2 \gamma} \int|\hat{\beta}(\xi-\eta)| \delta(\tau-|\eta|) \frac{1}{|\eta|^{2}}|\tilde{F}(|\eta|, \eta)|^{2} d \eta d \xi d \tau \\
& =\iint|\hat{\beta}(\xi-\eta)| \frac{|\eta|^{2 \gamma}}{|\eta|^{2}}|\tilde{F}(|\eta|, \eta)|^{2} d \eta d \xi \\
& \leq C \int \frac{|\eta|^{2 \gamma}}{|\eta|^{2}}|\tilde{F}(|\eta|, \eta)|^{2} d \eta \\
& \leq C\|F\|_{L_{t}^{r} L_{x}^{s}\left(\mathbb{R}^{1+n}\right)}^{2} .
\end{aligned}
$$

By a similar argument, we can show that the same bound holds for

$$
\int\left\|\beta(\cdot) \int \frac{e^{-i(t-s) \Lambda}}{\Lambda} F(s, \cdot) d s\right\|_{\dot{H}^{\gamma}\left(\mathbb{R}^{n}\right)}^{2} d t,
$$

which establishes (4.2). By duality, this is equivalent to having

$$
T^{*} F: L_{t}^{2} L_{x}^{2}\left(\mathbb{R}^{1+n}\right) \rightarrow L_{t}^{r^{\prime}} L_{x}^{s^{\prime}}\left(\mathbb{R}^{1+n}\right)
$$

bounded, where

$$
T^{*} F=\int \frac{\sin (s-t) \Lambda}{\Lambda} \beta(\cdot) \Lambda^{\gamma} F(s, \cdot) d s .
$$

We wanted to show, instead, that

$$
W F: L_{t}^{r} L_{x}^{s}\left(\mathbb{R}^{1+n}\right) \rightarrow L_{t}^{2} L_{x}^{2}\left(\mathbb{R}^{1+n}\right)
$$

is bounded, where

$$
W F(t, x)=\Lambda^{\gamma} \beta(\cdot) \int_{0}^{t} \frac{\sin (t-s) \Lambda}{\Lambda} F(s, \cdot) d s .
$$

By duality, this is equivalent to showing that

$$
W^{*} F: L_{t}^{2} L_{x}^{2}\left(\mathbb{R}^{1+n}\right) \rightarrow L_{t}^{r^{\prime}} L_{x}^{s^{\prime}}\left(\mathbb{R}^{1+n}\right)
$$

where

$$
W^{*} F(t, x)=\int_{t}^{\infty} \frac{\sin (s-t) \Lambda}{\Lambda} \beta(\cdot) \Lambda^{\gamma} F(s, \cdot) d s .
$$

This, however, follows from (4.2) after an application of the following lemma of Christ and Kiselev [2] (see also [12]).

Lemma 4.3. Let $X$ and $Y$ be Banach spaces and assume that $K(t, s)$ is a continuous function taking its values in $B(X, Y)$, the space of bounded linear mappings from $X$ to $Y$. Suppose that $-\infty \leq a<b \leq \infty$ and $1 \leq p<q \leq \infty$. Set

$$
T f(t)=\int_{a}^{b} K(t, s) f(s) d s
$$

and

$$
W f(t)=\int_{a}^{t} K(t, s) f(s) d s
$$


Suppose that

$$
\|T f\|_{L^{q}([a, b], Y)} \leq C\|f\|_{L^{p}([a, b], X)} .
$$

Then

$$
\|W f\|_{L^{q}([a, b], Y)} \leq C\|f\|_{L^{p}([a, b], X)} .
$$

\section{Strichartz ESTIMATES IN THE EXTERIOR DOMAIN}

By scaling, we may take $R=\frac{1}{2}$ in the sequel. We begin by proving a weighted version of Theorem 1.1 when the data and forcing terms are compactly supported.

Lemma 5.1. Suppose $n \geq 4$, and suppose $u$ is a solution to the Cauchy problem (1.1) with the forcing term $F$ replaced by $F+G$, where $F, G$ are supported in $\{|t| \leq 1\} \times\{|x| \leq 1\}$ and the initial data $f, g$ are supported in $\{|x| \leq 1\}$. Then, for admissible $p, q, r, s, \gamma$, there exists a positive, finite constant $C$ so that the following estimate holds for any $\theta<1$ :

$$
\begin{aligned}
& \left\|(|t|-|x|+2)^{\theta} u(t, x)\right\|_{L_{t}^{p} L_{x}^{q}(\mathbb{R} \times \Omega)} \\
& \quad \leq C\left(\|f\|_{\dot{H}_{D}^{\gamma}(\Omega)}+\|g\|_{\dot{H}_{D}^{\gamma-1}(\Omega)}+\|F\|_{L_{t}^{r} L_{x}^{s}(\mathbb{R} \times \Omega)}+\int\|G(t, \cdot)\|_{\dot{H}_{D}^{\gamma-1}(\Omega)} d t\right) .
\end{aligned}
$$

Proof. We will establish the result for $t \geq 0$. We begin this proof in the same manner as in Smith and Sogge [12. Start by observing that by (1.2) and Duhamel's principle, the result holds for $t \in[0,1]$, and by (1.2)

$$
\begin{aligned}
& \|u(1, \cdot)\|_{\dot{H}_{D}^{\gamma}(\Omega)}+\left\|\partial_{t} u(1, \cdot)\right\|_{\dot{H}_{D}^{\gamma-1}(\Omega)} \\
& \leq C\left(\|f\|_{\dot{H}_{D}^{\gamma}(\Omega)}+\|g\|_{\dot{H}_{D}^{\gamma-1}(\Omega)}+\|F\|_{L_{t}^{r} L_{x}^{s}(\mathbb{R} \times \Omega)}+\int\|G(t, \cdot)\|_{\dot{H}_{D}^{\gamma-1}(\Omega)} d t\right) .
\end{aligned}
$$

By considering $t \geq 1$, finite propagation speed and support considerations allow us to take $F=G=0$ with $f, g$ supported in $\{|x| \leq 2\}$.

We now fix a smooth $\beta$ with $\beta(x)=1$ for $|x| \leq \frac{1}{2}$, and $\beta(x)=0$ for $|x| \geq 1$. We then write $u$ as $u=\beta u+(1-\beta) u$ and will examine these pieces separately.

We begin by looking at $\beta u$. Notice that

$$
\square(\beta u)=\sum_{j=1}^{n} b_{j}(x) \partial_{x_{j}} u+c(x) u \equiv \tilde{G}(t, x)
$$

where $b_{j}, c$ are supported in $\frac{1}{2} \leq|x| \leq 1$. Since $|t|-|x| \leq|t|$, it will suffice to show

$$
\left\|(t+2)^{\theta} \beta(x) u(t, x)\right\|_{L_{t}^{p} L_{x}^{q}([1, \infty) \times \Omega)} \leq C\left(\|f\|_{\dot{H}_{D}^{\gamma}(\Omega)}+\|g\|_{\dot{H}_{D}^{\gamma-1}(\Omega)}\right) .
$$


By the local Strichartz estimate (1.2), Duhamel's principle, and local energy decay (2.1), we have

$$
\begin{aligned}
\|(t+2)^{\theta} & \beta(x) u(t, x) \|_{L_{t}^{p} L_{x}^{q}([1, \infty) \times \Omega)}^{p} \\
\leq & \sum_{j=1}^{\infty}(j+3)^{p \theta}\|\beta(x) u(t, x)\|_{L_{t}^{p} L_{x}^{q}([j, j+1] \times \Omega)}^{p} \\
\leq & C \sum_{j=1}^{\infty}(j+3)^{p \theta}\left(\|\beta(\cdot) u(j, \cdot)\|_{\dot{H}_{D}^{\gamma}(\Omega)}+\left\|\beta(\cdot) \partial_{t} u(j, \cdot)\right\|_{\dot{H}_{D}^{\gamma-1}(\Omega)}\right. \\
& \left.\quad+\int_{j}^{j+1}\|\tilde{G}(s, \cdot)\|_{\dot{H}_{D}^{\gamma-1}(\Omega)} d s\right)^{p} \\
\leq & C \sum_{j=1}^{\infty} \frac{(j+3)^{p \theta}}{(j+3)^{p(n / 2)}}\left(\|f\|_{\dot{H}_{D}^{\gamma}(\Omega)}+\|g\|_{\dot{H}_{D}^{\gamma-1}(\Omega)}\right)^{p} \\
& =C\left(\|f\|_{\dot{H}_{D}^{\gamma}(\Omega)}+\|g\|_{\dot{H}_{D}^{\gamma-1}(\Omega)}\right)^{p}
\end{aligned}
$$

as long as $\frac{n}{2}-\theta>\frac{1}{p}$. For $n \geq 4$, since $p \geq 2$, we have the above inequality provided $\theta<\frac{n-1}{2}$.

For the $v(t, x)=(1-\beta)(x) u(t, x)$ piece, we have that $v$ satisfies the Minkowski wave equation

$$
\left\{\begin{array}{l}
\square v(t, x)=-\tilde{G}(t, x), \\
v(0, x)=(1-\beta)(x) f(x), \\
\partial_{t} v(0, x)=(1-\beta)(x) g(x) .
\end{array}\right.
$$

Write $v=v_{0}+v_{1}$ where $v_{0}$ solves the homogeneous wave equation with the same Cauchy data as $v$, and $v_{1}$ solves the inhomogeneous wave equation with vanishing Cauchy data. Then, by Theorem 3.3, we have

$$
\begin{aligned}
\left\|(t-|x|+2)^{\theta}(1-\beta)(x) u(t, x)\right\|_{L_{t}^{p} L_{x}^{q}(\mathbb{R} \times \Omega)} & \\
\leq C\left(\|(1-\beta)(x) f\|_{\dot{H}^{\gamma}\left(\mathbb{R}^{n}\right)}+\right. & \left.\|(1-\beta)(x) g(x)\|_{\dot{H}^{\gamma-1}\left(\mathbb{R}^{n}\right)}\right) \\
& +\left\|(t-|x|+2)^{\theta} v_{1}(t, x)\right\|_{L_{t}^{p} L_{x}^{q}(\mathbb{R} \times \Omega)} .
\end{aligned}
$$

When $n \geq 4$, we can handle the last piece easily using local energy decay. By Duhamel's principle, write

$$
\left\|(t-|x|+2)^{\theta} v_{1}(t, x)\right\|_{L_{t}^{p} L_{x}^{q}(\mathbb{R} \times \Omega)}=\left\|(t-|x|+2)^{\theta} \int_{0}^{t} v_{1}(s ; t-s, x) d s\right\|_{L_{t}^{p} L_{x}^{q}(\mathbb{R} \times \Omega)}
$$

where $v_{1}(s ; \cdot, \cdot)$ solves

$$
\left\{\begin{array}{l}
\square v_{1}(s ; t, x)=0, \\
v_{1}(s ; 0, x)=0, \\
\partial_{t} v_{1}(s ; 0, x)=-\tilde{G}(s, x) .
\end{array}\right.
$$

Applying Minkowski's integral inequality, we have

$$
\left\|(t-|x|+2)^{\theta} v_{1}(t, x)\right\|_{L_{t}^{p} L_{x}^{q}(\mathbb{R} \times \Omega)} \leq C \int s^{\theta}\left\|(t-s-|x|+2)^{\theta} v_{1}(s ; t-s, x)\right\|_{L_{t}^{p} L_{x}^{q}(\mathbb{R} \times \Omega)} d s .
$$


Thus, by Theorem [3.3, the right side is bounded by

$$
\int s^{\theta}\|\tilde{G}(s, \cdot)\|_{\dot{H}^{\gamma-1}\left(\mathbb{R}^{n}\right)} d s .
$$

Finally, by local energy decay (2.1), we have that this is bounded by

$$
C \int s^{\theta-\frac{n}{2}}\left(\|f\|_{\dot{H}^{\gamma}(\Omega)}+\|g\|_{\dot{H}_{D}^{\gamma-1}(\Omega)}\right) d s \leq C\left(\|f\|_{\dot{H}^{\gamma}(\Omega)}+\|g\|_{\dot{H}_{D}^{\gamma-1}(\Omega)}\right)
$$

for $\theta<1$.

We are now ready to prove the main theorem.

Proof of Theorem 1.1. By the previous lemma, it will suffice to show the result when $f$ and $g$ vanish for $\{|x| \leq 1\}$.

We begin by decomposing $u$ into

$$
u(t, x)=u_{0}(t, x)-v(t, x)
$$

where $u_{0}$ solves the Minkowski wave equation

$$
\left\{\begin{array}{l}
\square u_{0}(t, x)=F(t, x), \\
u_{0}(0, x)=f(x), \\
\partial_{t} u_{0}(0, x)=g(x) .
\end{array}\right.
$$

Here $F$ is assumed to be 0 on $\mathbb{R}^{n} \backslash \Omega$.

We now fix a smooth compactly supported $\beta$ such that $\beta(x)=1$ for $|x| \leq 1 / 2$ and $\beta(x)=0$ for $x \geq 1$. Then, further decompose $u$ into

$$
u(t, x)=u_{0}(t, x)-v(t, x)=(1-\beta)(x) u_{0}(t, x)+\left(\beta(x) u_{0}(t, x)-v(t, x)\right) .
$$

By the global Minkowski Strichartz estimate (1.3), $(1-\beta)(x) u_{0}(t, x)$ satisfies the desired estimate. Thus, we may focus on $\beta(x) u_{0}(t, x)-v(t, x)$.

We have that $\beta(x) u_{0}(t, x)-v(t, x)$ satisfies

$$
\square\left(\beta(x) u_{0}(t, x)-v(t, x)\right)=\beta(x) F(t, x)+G(t, x)
$$

with zero Cauchy data (since we are assuming that $f, g$ vanish for $|x| \leq 1$ ). Here

$$
G(t, x)=\sum_{j=1}^{n} b_{j}(x) \partial_{x_{j}} u_{0}(t, x)+c(x) u_{0}(t, x)
$$

where $b_{j}, c$ vanish for $|x| \geq 1$. By Lemma 4.2

$$
\int_{-\infty}^{\infty}\|G(t, \cdot)\|_{\dot{H}_{D}^{\gamma-1}(\Omega)}^{2} d t \leq C\left(\|f\|_{\dot{H}_{D}^{\gamma}(\Omega)}+\|g\|_{\dot{H}_{D}^{\gamma-1}(\Omega)}+\|F\|_{L_{t}^{r} L_{x}^{s}(\mathbb{R} \times \Omega)}\right)^{2} .
$$

Let

$$
\begin{aligned}
F_{j}(t, x) & =\chi_{[j, j+1]}(t) F(t, x), \\
G_{j}(t, x) & =\chi_{[j, j+1]}(t) G(t, x),
\end{aligned}
$$

and write (for $t>0$ )

$$
\beta u_{0}-v=\sum_{j=0}^{\infty} u_{j}(t, x)
$$


where $u_{j}(t, x)$ is the forward solution to

$$
\square u_{j}(t, x)=\beta(x) F_{j}(t, x)+G_{j}(t, x)
$$

with zero Cauchy data.

Thus, by Lemma 5.1 we have

$$
\begin{aligned}
& \left\|(t-j-|x|+2)^{\theta} u_{j}(t, x)\right\|_{L_{t}^{p} L_{x}^{q}(\mathbb{R} \times \Omega)} \\
& \quad \leq C\left(\left\|F_{j}(t, x)\right\|_{L_{t}^{r} L_{x}^{s}(\mathbb{R} \times \Omega)}+\int_{j}^{j+1}\|G(t, \cdot)\|_{\dot{H}_{D}^{\gamma-1}(\Omega)} d t\right) .
\end{aligned}
$$

Since $u_{j}$ is supported in the region $t-j-|x|+2 \geq 1$, an application of the Cauchy-Schwartz inequality yields

$$
\begin{aligned}
& \left|\beta(x) u_{0}(t, x)-v(t, x)\right| \leq \sum_{j=0}^{\infty}\left|u_{j}(t, x)\right| \\
& \leq\left(\sum_{j=0}^{\infty}(t-j-|x|+2)^{-2 \theta}\right)^{1 / 2}\left(\sum_{j=0}^{\infty}\left[(t-j-|x|+2)^{\theta} u_{j}(t, x)\right]^{2}\right)^{1 / 2} \\
& \quad \leq C\left(\sum_{j=0}^{\infty}\left[(t-j-|x|+2)^{\theta} u_{j}(t, x)\right]^{2}\right)^{1 / 2}
\end{aligned}
$$

since we can choose $\theta>1 / 2$.

Since $1 \leq r, s \leq 2 \leq p, q$, Minkowski's integral inequality, (15.2), and (15.3) yield

$$
\begin{aligned}
\| \beta(x) u_{0}(t, x) & -v(t, x) \|_{L_{t}^{p} L_{x}^{q}(\mathbb{R} \times \Omega)}^{2} \\
& \leq C \sum_{j=0}^{\infty}\left\|(t-j-|x|+2)^{\theta} u_{j}\right\|_{L_{t}^{p} L_{x}^{q}(\mathbb{R} \times \Omega)}^{2} \\
& \leq C \sum_{j=0}^{\infty}\left\|F_{j}\right\|_{L_{t}^{r} L_{x}^{s}(\mathbb{R} \times \Omega)}^{2}+C \sum_{j=0}^{\infty}\left(\int_{j}^{j+1}\|G(t, \cdot)\|_{\dot{H}_{D}^{\gamma-1}(\Omega)} d t\right)^{2} \\
& \leq C \sum_{j=0}^{\infty}\left\|F_{j}\right\|_{L_{t}^{r} L_{x}^{s}(\mathbb{R} \times \Omega)}^{2}+C \sum_{j=0}^{\infty}\left(\int_{j}^{j+1}\|G(t, \cdot)\|_{\dot{H}_{D}^{\gamma-1}(\Omega)}^{2} d t\right) \\
& \leq C\|F\|_{L_{t}^{r} L_{x}^{s}(\mathbb{R} \times \Omega)}^{2}+C \int_{0}^{\infty}\|G(t, \cdot)\|_{\dot{H}_{D}^{\gamma-1}(\Omega)}^{2} d t \\
& \leq C\left(\|f\|_{\dot{H}_{D}^{\gamma}(\Omega)}+\|g\|_{\dot{H}_{D}^{\gamma-1}(\Omega)}+\|F\|_{L_{t}^{r} L_{x}^{s}(\mathbb{R} \times \Omega)}\right)^{2},
\end{aligned}
$$

as desired.

\section{REFERENCES}

[1] N. Burq: Global Strichartz estimates for nontrapping geometries: A remark about an article by H. Smith and C. Sogge, Comm. Partial Differential Equations, 28, (2003), no. 9-10, 1675-1683. MR 2001179 (2004g:35146)

[2] M. Christ, A. Kiselev: Maximal functions associated to filtrations, J. Funct. Anal., $\underline{\mathbf{1 7 9}}$, (2001), 409-425. MR 1809116 (2001i:47054)

[3] L. Hörmander: Lectures on Nonlinear Hyperbolic Differential Equations, Springer-Verlag, 1997. MR 1466700 (98e:35103) 
[4] M. Keel, T. Tao: Endpoint Strichartz Estimates, Amer J. Math., 120, (1998), 955-980. MR 1646048 (2000d:35018)

[5] P. D. Lax, R. S. Philips: Scattering Theory (Revised Edition), Academic Press Inc., 1989. MR 1037774 (90k:35005)

[6] R. Melrose: Singularities and energy decay in acoustical scattering, Duke Math. J., $\underline{\mathbf{4 6}}$, (1979), 43-59. MR 0523601 (80h:35104)

[7] C. Morawetz: Decay for solutions of the exterior problem for the wave equation, Comm. Pure Appl. Math., 르, (1975), 229-264. MR 0372432 (51:8641)

[8] C. Morawetz: The decay of solutions of the exterior initial-boundary value problem for the wave equation, Comm. Pure Appl. Math., 14, (1961), 561-568. MR 0132908 (24:A2744)

[9] C. Morawetz: Exponential Decay of Solutions of the Wave Equation, Comm. Pure Appl. Math., 19, (1966), 439-444. MR 0204828 (34:4664)

[10] C. Morawetz, J. Ralston, W. Strauss: Decay of solutions of the wave equation outside nontrapping obstacles, Comm. Pure Appl. Math., $\underline{\mathbf{3 0}}$, (1977), 447-508. MR 0509770 (58:23091a)

[11] J. Ralston: Note on the decay of acoustic waves, Duke Math. J., $\underline{46}$, (1979), 799-804. MR 0552527 (80m:35051)

[12] H. Smith, C.D. Sogge: Global Strichartz estimates for nontrapping perturbations of the Laplacian, Comm. Partial Differential Equations, 2 $\underline{\mathbf{5}}$, (2000), 2171-2183. MR 1789924 (2001j:35180)

[13] H. Smith, C.D. Sogge: On the critical semilinear wave equation outside convex obstacles, J. Amer. Math. Soc., $\underline{\mathbf{8}}$, (1995), 879-916. MR 1308407 (95m:35128)

[14] W. Strauss: Dispersal of waves vanishing on the boundary of an exterior domain, Comm. Pure Appl. Math., $\underline{\mathbf{2 8}}$, (1975), 265-278. MR 0367461 (51:3703)

[15] R. Strichartz: A priori estimates for the wave equation and some applications, J. Funct. Analysis, $\underline{\mathbf{5}}$, (1970), 218-235. MR 0257581 (41:2231)

[16] R. Strichartz: Restriction of Fourier transform to quadratic surfaces and decay of solutions to the wave equation, Duke Math. J., $\underline{\mathbf{4 4}}$, (1977), 705-714. MR 0512086 (58:23577)

[17] M. Taylor: Grazing rays and reflection of singularities of solutions to wave equations, Comm. Pure Appl. Math., $\underline{\mathbf{2 9}}$, (1976), 1-38. MR 0397175 (53:1035)

[18] M. Taylor: Partial differential equations I, Springer-Verlag, Berlin, 1996. MR 1395148 (98b:35002b)

[19] B. R. Vainberg: On the short wave asymptotic behavior of solutions of stationary problems and the asymptotic behavior as $t \rightarrow \infty$ of solutions of non-stationary problems, Russian Math Surveys, 30:2, (1975), 1-55. MR 0415085 (54:3176)

School of Mathematics, Georgia Institute of Technology, Atlanta, Georgia 30332

E-mail address: metcalfe@math.gatech.edu 